\section{Sistema de Informações sobre Mortalidade: estudo de caso em municípios com precariedade dos dados}

\author{
Mortality Data System: a case study on \\ municipalities with data underreporting
}

\begin{abstract}
The study was part of a survey conducted in clusters of Brazilian municipalities (counties) characterized by serious underreporting of deaths, with the objective of estimating infant mortality in these areas in 2000. The article discusses the principal sources of information on infant deaths in these municipalities, as well as some problems related to the implementation of the Mortality Data System (SIM). The methodology included an active search for deaths in previously registered official and unofficial sources, which identified 344 deaths not processed in the SIM (66\% of total deaths). There were a low percentage of deaths processed in the SIM and coming from official sources (notary public offices and healthcare facilities), thus highlighting problems with reporting of deaths, issuing of death certificates, and data flows. The important contribution by the Community Health Agents Program/ Family Health Program points to the need for active data search in this source as a routine activity in municipalities with underreporting of data. Insufficient training of professionals and high staff turnover were major issues in the system's deficient implementation.
\end{abstract}

Information Systems; Infant Mortality; Family Health Program
Paulo Germano de Frias 1

Pricila Melissa Honorato Pereira 1

Carla Lourenço Tavares de Andrade 2

Célia Landmann Szwarcwald 3

\section{Introdução}

O Sistema de Informações sobre Nascidos Vivos (SINASC) e o Sistema de Informações sobre Mortalidade (SIM) foram criados pelo Ministério da Saúde com o objetivo de compilar os dados de nascimentos e óbitos ocorridos no território nacional e permitir a construção de indicadores demográficos e de saúde da população.

A existência de um documento padronizado para a coleta de dados sobre nascidos vivos, a Declaração de Nascido Vivo (DNV), permite estabelecer e comparar as características sóciodemográficas e assistenciais das gestantes, bem como as condições do nascimento nas distintas regiões brasileiras ${ }^{1}$. A cobertura do SINASC tem aumentado desde a sua implantação e teve, em 2004 , abrangência de cerca de $87 \%$ segundo os dados divulgados pela Rede Interagencial de Informações para a Saúde (RIPSA) 2 .

Já o SIM, implantado em 1976 3, tem ainda precariedade dos dados em alguns municípios nas regiões Norte e Nordeste, principalmente no que se refere à qualidade das informações 4 . As lacunas das informações nos municípios mais pobres mostram as enormes iniqüidades em saúde da população brasileira, refletindo a falta de acesso aos bens e serviços públicos.

No que se refere à construção de indicadores de saúde por meio das informações do Ministério da Saúde, como o coeficiente de mortalidade infantil, a precariedade dos dados em algumas 
áreas do país não permite a utilização do método direto de cálculo. Por isso, em alguns estados do Brasil, considerados como de baixa qualidade da informação, persiste o uso de estimativas indiretas de mortalidade calculadas pelo Instituto Brasileiro de Geografia e Estatística (IBGE) para orientar as políticas públicas 2 .

Considerando as limitações inerentes ao uso de estimativas indiretas já comentadas por outros autores 5,6 associadas ao desestímulo aos profissionais e gestores de saúde pela impossibilidade de monitorar a diminuição dos óbitos infantis 7, o Ministério da Saúde vem adotando medidas em busca da melhoria da qualidade da informação. Em 2001, por solicitação do Ministério da Saúde, a Fundação Oswaldo Cruz e o Instituto Materno Infantil Professor Fernando Figueira realizaram uma pesquisa em municípios das regiões Norte e Nordeste, visando à estimação da mortalidade infantil no ano 2000, de maneira direta 8 . O método adotado foi o da busca ativa de óbitos em aglomerados de municípios classificados entre aqueles com grande deficiência das informações vitais.

A etapa de campo para identificação de óbitos não notificados ao sistema permitiu conhecer um conjunto de problemas na operacionalização do SIM. O presente artigo tem como objetivo sistematizar e discutir esses aspectos observados no processo de busca ativa de óbitos e o comportamento da notificação em anos imediatamente posteriores à pesquisa de campo.

\section{Metodologia}

Na primeira etapa do estudo foram utilizados critérios de adequação da informação do SIM e do SINASC para identificar municípios com precariedade dos dados, considerando o período 1996-1998. Os critérios para classificação dos municípios como satisfatórios para as informações de nascimento e óbito foram os propostos por Szwarcwald et al. 5 .

Nos estados que apresentaram grande deficiência das informações vitais, foi realizado um processo de busca ativa de óbitos em aglomerados homogêneos de municípios selecionados, com o objetivo de subsidiar o cálculo de estimativas de mortalidade infantil nas áreas com informação não satisfatória.

Para a obtenção de um número suficientemente grande de óbitos que possibilitasse a estimação da mortalidade infantil, estimou-se que cada conglomerado de municípios teria que ter pelo menos 50 mil habitantes. Sendo assim, foram escolhidos municípios vizinhos (ou próximos) com grande deficiência das informa- ções vitais, formando aglomerados de mais de 50 mil habitantes. Uma outra condição era que esses municípios fossem cobertos pelo Programa Agentes Comunitários de Saúde/Programa Saúde da Família (PACS/PSF), para que as informações do Sistema de Informações da Atenção Básica (SIAB) pudessem ser validadas.

Foram escolhidos oito aglomerados de municípios nos estados do Amazonas, Pará, Maranhão, Paraíba, Piauí e Bahia. Os municípios selecionados para a pesquisa foram: Iranduba e Nova Olinda do Norte (aglomerado 1, Amazonas); Barcarena (aglomerado 2, Pará); Vigia e Santo Antônio do Tauá (aglomerado 3, Pará); Nossa Senhora dos Remédios, Cabeceiras do Piauí e Barras (aglomerado 4, Piauí); Codó (aglomerado 5, Maranhão); Cabedelo e Pedras de Fogo (aglomerado 6, Paraíba); Ribeira do Pombal e Ribeira do Amparo (aglomerado 7, Bahia); Tancredo Neves, Laje e Teolândia (aglomerado 8, Bahia).

A coleta de dados envolveu os óbitos fetais e não fetais de menores de um ano de residentes nos municípios selecionados, ocorridos entre os dias 1 o de janeiro e 31 de dezembro de 2000. A finalidade da incorporação dos óbitos fetais no universo da coleta foi de possibilitar a posterior validação do tipo de óbito.

As etapas iniciais consistiram na listagem nominal de todos os óbitos fetais e não fetais de menores de um ano, residentes nos municípios selecionados, a partir de dados disponibilizados pelo Ministério da Saúde, com posterior busca nas Secretarias Estaduais e Municipais de Saúde.

A coleta de dados utilizou instrumento padronizado e objetivou a identificação tanto de Declarações de Óbito (DO) emitidas e não informadas ao SIM como de óbitos que não geraram as respectivas DO. Foram utilizadas as seguintes fontes de informações: cartórios; cemitérios oficiais e não oficiais (livros de registro, guias de sepultamento, originais de DO); estabelecimentos de saúde (livros de estatísticas hospitalares, de controle de admissão e alta e da sala de parto, guias de Autorização de Internação Hospitalar - AIH e, prontuários); agentes comunitários de saúde, profissionais do PSF (informações escritas na ficha D do SIAB e verbais); informantes-chave como parteiras tradicionais, líderes comunitários, curandeiros, rezadeiras, além de funerárias e farmácias. Foram utilizadas múltiplas fontes com o intuito de verificar a confiabilidade das informações coletadas.

Em se tratando de uso de dados secundários, coletados nas fontes de notificação, para realização da pesquisa de campo, o Ministério da Saúde solicitou às Secretarias Municipais e Estaduais de Saúde, através de carta, o consen- 
timento para uso e cadastramento das fontes de possível obtenção dos dados.

Após a busca ativa, os óbitos coletados no nível local foram disponibilizados às Secretarias Estaduais e Municipais de Saúde, que após confrontar as informações coletadas no nível local com as informadas ao SIM, foram orientadas a emitir e processar as respectivas DO, como recomendado pelo Ministério da Saúde (DO com fim estatístico) 9 .

Os problemas identificados na operacionalização do SIM foram sistematizados de acordo com os componentes do modelo de operacionalização do sistema, construído com base na normatização vigente 10 , e adaptado à realidade dos municípios estudados (Figura 1). A discussão acerca dos problemas identificados buscou seguir a organização do modelo de operacionalização do SIM formado por seis componentes: notificação, coleta e processamento de dados, análise dos dados, busca ativa em fontes alternativas, divulgação das informações, normatização e fluxos entre as esferas de gestão do SIM.

A análise estatística dos dados consistiu em identificar as principais fontes de notificação e estimar a cobertura de óbitos informados e compilados no SIM, por região. Também foi observada e comparada a média de óbitos infantis nos aglomerados de municípios em dois quadriênios (1996-1999) antes e (2001-2004) depois da pesquisa de campo. Para isso, foram utilizados os dados do SIM do Ministério da Saúde, disponibilizados por meio da página da Internet do Departamento de Informática do SUS (DATASUS; http://www.datasus.gov.br). Esses dados foram coletados no ano de 2007 e a comparação teve como objetivo verificar a captação de óbitos nos anos posteriores à pesquisa.

Figura 1

Modelo de operacionalização do Sistema de Informações sobre Mortalidade (SIM) em municípios com precariedade dos dados.

Componentes
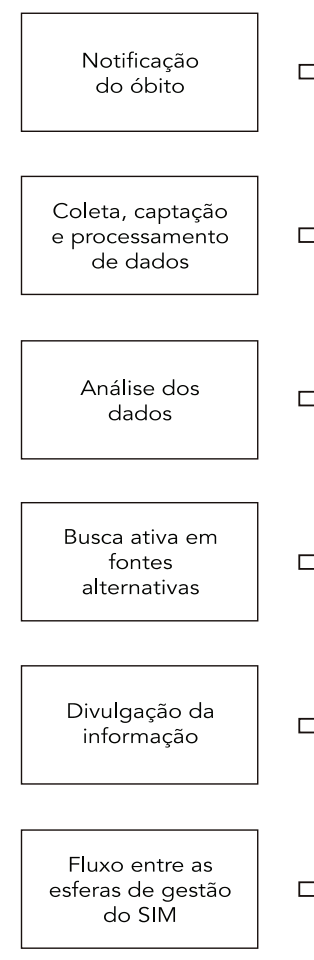

Atividades

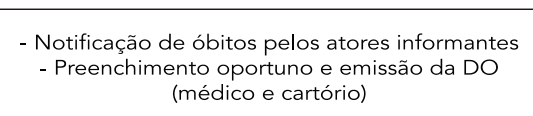
(médico e cartório)

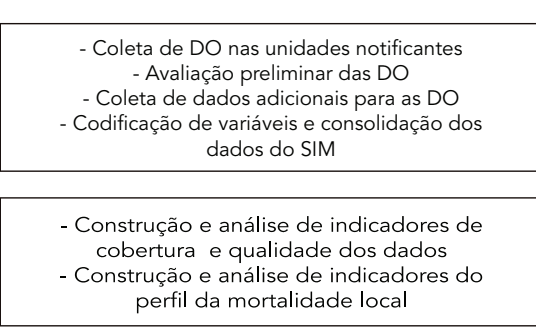
- Coleta de DO e informações em fontes não oficiais (cemitérios, unidades de saúde da família e através de informantes-chave) - Entrada de dados adicionais no SIM

\section{- Divulgação das informações por meio de boletins simplificados para as áreas técnicas, estabelecimentos de saúde, entidades de controle social}

- Distribuição de insumos e normas (esfera estadual-municipal)

- Envio de dados (esfera municipal-estadual)

\section{- Atores informantes conhecedores do fluxo de notificação habilitados e sensibilizados para o preenchimento da DO}
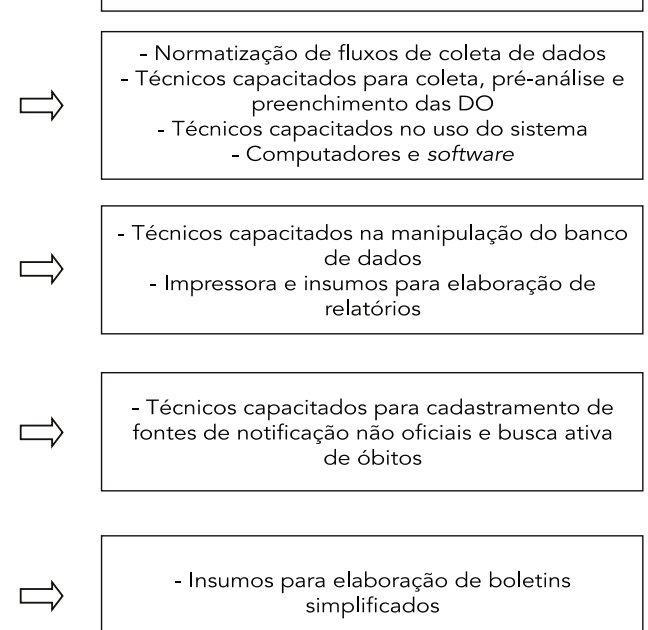
simplificados

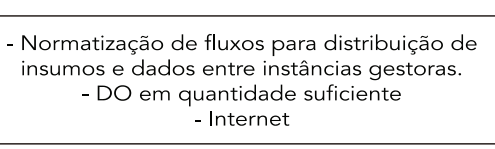




\section{Resultados}

O processo de busca ativa identificou 344 óbitos infantis não processados no SIM, cerca de $66 \%$ do total de óbitos infantis de residentes no conjunto de oito aglomerados de municípios selecionados para o estudo. Esse percentual variou entre $42,3 \%$ e $78,4 \%$ entre os conglomerados como observado na Tabela 1. Na Região Norte como um todo o percentual de óbitos não processados pelo SIM foi maior (Tabela 1).

Observando-se a contribuição da pesquisa sob o aspecto da continuidade dos resultados alcançados na melhoria da cobertura do SIM, foram comparadas as médias dos óbitos notificados ao SIM nos quatro anos anteriores e posteriores à pesquisa (Tabela 1). Mesmo com a queda observada quando comparado ao ano 2000 e à média de óbitos do quadriênio 2001-2004, foi percebida melhoria em relação ao quadriênio 1996-1999. Entre os oito aglomerados pesquisados, três (dois na Região Norte e um na Região Nordeste) apresentaram incremento percentual acima de $100 \%$. Outros quatro tiveram incrementos gradativamente menores e um teve redução proporcional da média de óbitos infantis notificados.

Os dados apresentados na Tabela 1 mostram ainda que houve uma diferença entre o número de óbitos captados pelo processo de busca ativa (520) e o número de óbitos disponibilizado no site do DATASUS (476), provavelmente porque os municípios deixaram de emitir as DOs correspondentes. Outra explicação possível seria dada pelas perdas no processo de transferência dos dados.

Analisando-se a contribuição das principais fontes de notificação pesquisadas (Tabela 2), destacaram-se, na Região Norte: estabelecimentos de saúde, cemitério e PACS/PSF. As fontes de informação em geral tiveram um baixo percentual de óbitos processados pelo SIM nessa região. As fontes oficiais de notificação do óbito "estabelecimentos de saúde" e "cartório" chamam a atenção pelo baixo percentual de óbitos processados pelo SIM (respectivamente $31,9 \%$ e $22,2 \%$ ), apesar de terem sido estes os maiores percentuais quando consideradas as demais fontes. Entre os óbitos identificados através da fonte PACS/PSF (39 óbitos), apenas 5,1\% estavam processadas no SIM.

Na Região Nordeste (Tabela 2), o PACS/PSF constituiu a principal fonte para a identificação de óbitos infantis para a pesquisa (184 óbitos - $52 \%$ do total). Os estabelecimentos de saúde também foram uma importante fonte de notificação na Região Nordeste, captando-se cerca de $30 \%$ do total de óbitos. Mas ao contrário da

Tabela 1

Número de óbitos infantis nos aglomerados de municípios segundo processamento ou não no Sistema de Informações sobre Mortalidade (SIM), número de óbitos informados após a pesquisa no ano de 2000 e média de óbitos infantis nos quadriênios 1996-1999 e 2001-2004

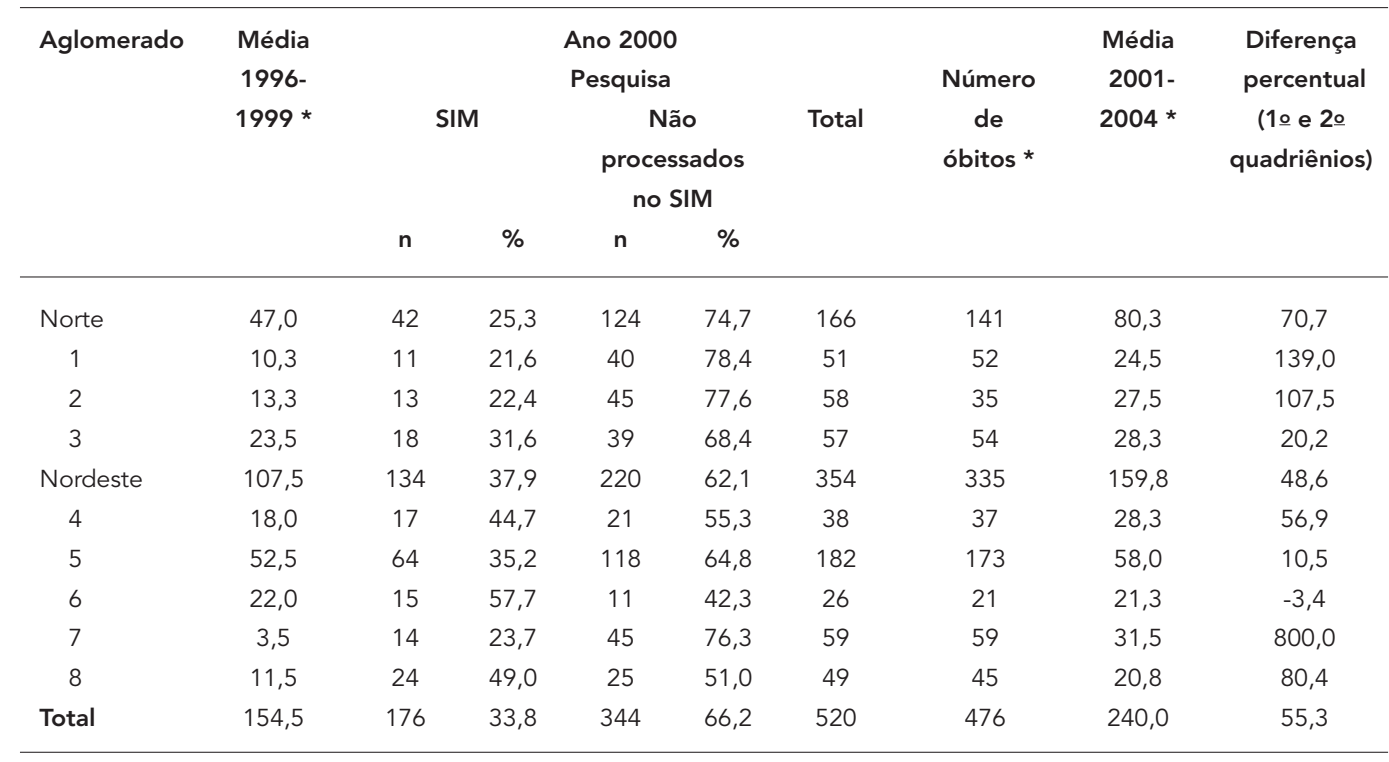

* Dados contidos no banco de dados do Ministério da Saúde, disponibilizados pelo Departamento de Informática do SUS (DATASUS; http://www.datasus.gov.br). 
Região Norte, grande parte já estava processada no SIM antes da realização da pesquisa.

$\mathrm{Na}$ Tabela 3, as fontes de informações foram classificadas como "fora" ou "dentro do sistema de saúde", sendo esta última categoria representada por PACS/PSF e estabelecimentos de saúde, enquanto a primeira compreendeu as demais fontes. A análise segundo esta classificação mostrou que a maioria dos óbitos encontrados estava disponível dentro do sistema de saúde. Apesar disso, uma pequena proporção desses óbitos foi informada ao SIM.

A sistematização dos problemas identificados na operacionalização do SIM permitiu a organização destes de acordo com os componentes do sistema descritos no método, como pode ser visto na Figura 2.

Esses problemas foram verificados em várias etapas do processo de informação do óbito infantil. Caracterizam-se, principalmente, por erros na notificação do óbito infantil, mesmo os ocorridos dentro do sistema de saúde, pela ausência de estratégias sistemáticas para busca ativa de óbitos, por problemas no fluxo de instrumentos, na digitação e análise dos dados. Como dimensão transversal a todas as outras, destacaram-se os seguintes aspectos: ausência de mecanismos de sensibilização e capacitação dos profissionais de saúde; insuficiência de supervisão; e a alta rotatividade de técnicos para o gerenciamento da informação em nível municipal.

\section{Discussão}

A existência de sistemas de informações acessíveis e confiáveis na esfera municipal é condição essencial para a elaboração do diagnóstico,

Tabela 2

Freqüência absoluta e percentual de óbitos identificados segundo fonte notificadora e percentual de óbitos captados pelo Sistema de Informações sobre Mortalidade (SIM). Regiões Norte e Nordeste do Brasil, 2000.

\begin{tabular}{|c|c|c|c|c|}
\hline $\begin{array}{l}\text { Região dos municípios } \\
\text { pesquisados }\end{array}$ & Fonte & $\begin{array}{c}\text { Número de } \\
\text { óbitos }\end{array}$ & $\begin{array}{l}\text { Percentual no } \\
\text { total de óbitos* }\end{array}$ & $\begin{array}{l}\text { Percentual de óbitos } \\
\text { captados pelo SIM }\end{array}$ \\
\hline \multirow[t]{5}{*}{ Norte } & Domicílio & 28 & 16,9 & 17,9 \\
\hline & Cartório & 18 & 10,8 & 22,2 \\
\hline & Cemitério & 59 & 35,5 & 20,3 \\
\hline & PACS/PSF & 39 & 23,5 & 5,1 \\
\hline & ecimentos de saúde & 69 & 41,6 & 31,9 \\
\hline \multirow[t]{5}{*}{ Nordeste } & Domicílio & 33 & 9,3 & 33,3 \\
\hline & Cartório & 19 & 5,4 & 78,9 \\
\hline & Cemitério & 14 & 4,0 & 42,9 \\
\hline & PACS/PSF & 184 & 52,0 & 16,3 \\
\hline & ecimentos de saúde & 100 & 28,2 & 70,0 \\
\hline
\end{tabular}

PACS/PSF: Programa Agentes Comunitários de Saúde/Programa Saúde da Família.

* A soma não totaliza $100 \%$ porque um mesmo óbito pode ter sido encontrado em mais de uma fonte pesquisada.

Tabela 3

Número de óbitos identificados segundo a fonte notificadora (dentro ou fora do sistema de saúde) e percentual de óbitos captados pelo Sistema de Informações sobre Mortalidade (SIM). Regiões Norte e Nordeste do Brasil, 2000.

\begin{tabular}{|c|c|c|c|c|}
\hline \multirow[t]{2}{*}{ Tipo de fonte de informações } & \multicolumn{2}{|c|}{ Norte } & \multicolumn{2}{|c|}{ Nordeste } \\
\hline & $\mathrm{n}$ & Percentual no SIM & $\mathbf{n}$ & Percentual no SIM \\
\hline Dentro do sistema de saúde & 103 & 22,3 & 264 & 32,6 \\
\hline \multicolumn{5}{|l|}{ (PACS/PSF ou estabelecimento de saúde) } \\
\hline Fora do sistema de saúde & 63 & 30,2 & 90 & 53,3 \\
\hline
\end{tabular}

PACS/PSF: Programa Agentes Comunitários de Saúde/Programa Saúde da Família. 
Problemas identificados durante a busca ativa de declarações de óbito de acordo com o modelo de operacionalização do Sistema de Informações sobre Mortalidade (SIM) em municípios do Norte e Nordeste do Brasil com precariedade dos dados.

Componentes do modelo de operacionalização do SIM
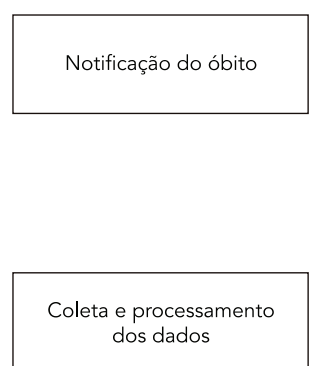

Análise de dados
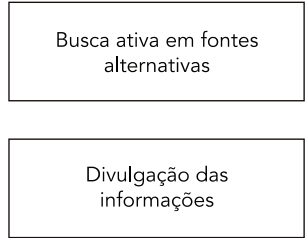

Normatização e fluxos
entre esferas de gestão do SIM
Problemas identificados durante a busca ativa

Em cada componente

Não notificação de óbitos identificados por agentes comunitários de saúde ou outros informantes com conseqüente não emissão da DO

Ausência de estratégias para as ocorrências de óbitos domiciliares da zona rural, onde os cartórios praticamente não registram óbitos de menores de um ano;

Não emissão de DO para óbitos ocorridos em estabelecimentos de saúde

Uso indevido de termos como aborto, óbito fetal, óbito não fetal, gerando não emissão de instrumentos;

Preenchimento de DO por agentes administrativos, auxiliares de enfermagem etc.

DO emitidas sem identificação do falecido.

Ausência ou fragilidade das atividades relacionadas à busca ativa de DO em Estabelecimentos de Saúde cartórios e centrais de registro:

Desconhecimento ou pouca compreensão dos fluxos da DO

Ausência ou fragilidade nas rotinas de verificação da consistência dos dados:

- Precárias ou inadequadas codificação e digitação de variáveis demográficas.

- Ausência de parâmetros de identificação de sub-notificação e flutuações de dados:

Precária análise dos dados na construção de indicadores de saúde.

\section{- Ausência de estratégias para captação de óbitos em áreas rurais ou com precário acesso ao sistema} oficial de notificação, utilizando fontes alternativas como cemitérios, PACS/PSF ou parteiras tradicionais.

\section{- Ausência ou precária retroalimentação dos dados para as unidades notificantes;} - Ausência de práticas de divulgação das informações.

Circulação de duas versões de DO (inadequado recolhimento da versão antiga);

- Utilização de instrumento não padronizado, produzido e impresso no município

- Distribuição de instrumentos das SES para as SMS e destas para as unidades de saúde baseada apenas na quantidade de notificações recebidas;

Problemas no fluxo e transferência dos dados (óbitos digitados na SMS ou SES transferidos e que não aparecem no banco nacional e vice-versa).
Comuns a todos os componentes

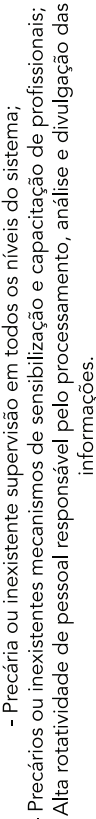

DO: Declaração de Óbito; PACS/PSF: Programa Agentes Comunitários de Saúde/Programa Saúde da Família; SES: Secretaria Estadual de Saúde; SMS: Secretaria Municipal de Saúde.

planejamento e programação de ações efetivas na melhoria da situação de saúde. Nesse sentido, a sub-notificação de eventos vitais é um sério obstáculo ao conhecimento de importantes indicadores epidemiológicos, limitando o uso dos dados provenientes dos sistemas de informação na maioria dos estados brasileiros 11 .

Em virtude das desigualdades existentes relativas à notificação dos eventos vitais, faz-se necessário o conhecimento do contexto onde esses dados são produzidos, o município. Uma das estratégias utilizadas é o estudo de caso, indicado quando o investigador tem pouco controle sobre os acontecimentos, pela observação do fenômeno a partir do interior dos casos possibilitando uma análise das questões em profundidade 12,13,14. Essa estratégia de pesquisa envolve uma ampla variedade de evidências - documentos, artefatos, entrevistas e observações - para compor o cenário no qual se recorta o objeto de estudo.

A primeira parte dos resultados apresentados revelou a baixa cobertura do SIM nos aglomerados selecionados. A busca ativa de óbitos tem sido praticada por alguns pesquisadores para identificar eventos não notificados, ou mesmo, desconhecidos do sistema de saúde, a fim de melhorar a cobertura dos dados do SIM 15,16,17,18,19,20,21.

Os resultados do presente estudo ressaltaram o potencial de algumas dessas fontes, corroborando os achados de outros estudos que utilizaram a estratégia da busca ativa. Estudo de Frias et al. 22 em um município do Nordeste destacou a contribuição dos estabelecimentos de saúde na recuperação de óbitos hospitalares e dos agentes comunitários no caso de óbitos domiciliares. 
O baixo percentual de óbitos processados no SIM provenientes das fontes oficiais: cartório - principalmente na Região Norte - e hospital estão relacionados tanto a problemas no registro/notificação dos óbitos, resultando na não emissão da DO, quanto no fluxo de DO já emitidas, questões levantadas no estudo. Por outro lado, dificuldades de acesso a serviços de saúde e cartórios estabelecem a importância da pesquisa de óbitos em fontes alternativas que tiveram participação considerável na fatia de óbitos detectados.

No modelo de operacionalização do SIM, o objetivo final é a produção e divulgação de informação de qualidade para subsidiar as políticas de saúde. O componente "busca ativa em fontes alternativas" foi incluído no modelo para os municípios com precariedade dos dados considerando a importância que estas fontes tiveram na captação de óbitos nessas localidades.

Em relação ao componente "notificação do óbito", foram identificados vários problemas que já haviam sido citados por outros autores, tais como: a incorreta classificação de óbitos fetais e neonatais; o inadequado preenchimento de variáveis da DO; a não emissão das declarações dentro de estabelecimentos de saúde 21,22,23.

Já no que diz respeito à coleta dos dados, segundo Almeida et al. 23, em muitos municípios brasileiros, as DO são coletadas apenas nos cartórios de registro civil, apesar da ênfase existente para que essas sejam complementadas com busca ativa nos serviços de saúde 22,24 .

O baixo percentual de informação ao SIM das mortes infantis encontradas em fontes integrantes do sistema de saúde aponta para a necessidade de fortalecimento das ações de notificação e coleta de DO nos estabelecimentos de saúde bem como o resgate da informação entre as equipes de saúde da família (e/ou agentes comunitários). O PACS/PSF tem desempenhado um importante papel, principalmente com a expansão de cobertura da atenção básica em saúde desde a década de 1990. O estudo dessas fontes vem confirmando sua importância na melhoria da qualidade dos dados 16,17,18,22. Iniciativas têm sido realizadas no sentido de melhorar a adesão desses profissionais à notificação de eventos vitais. Entre eles está a construção da Cartilha dos Sistemas de Informação sobre Nascidos Vivos e Mortalidade para as equipes de saúde da família 24 . Assim, enfatiza-se a importância das atividades de gestão do sistema nos níveis regional, estadual e municipal, que podem contribuir para a melhor articulação das áreas de assistência à saúde e vigilância.

Para áreas com baixa cobertura dos serviços de saúde, especialmente na zona rural, a estra- tégia de captação dos óbitos a partir do registro civil via cartórios tem se mostrado insuficiente $22,25,26$, o que corrobora os resultados encontrados. O sub-registro de eventos vitais é maior justamente nessas áreas, entre outros motivos, pelo não reconhecimento da importância do documento 22,26. No caso do óbito infantil, isso é ainda mais acentuado, sendo freqüentes os sepultamentos ilegais, sem conseqüente emissão da certidão de óbito 20,22. Estudos realizados em comunidades rurais na África do Sul e México revelaram o pouco conhecimento sobre a importância do registro do óbito infantil entre as mães entrevistadas, assim como a falta de clareza sobre os procedimentos para o registro. A maior distância do cartório e o menor porte populacional das comunidades foram fatores relacionados a um maior percentual de sub-registro 27,28 .

Quanto ao processamento dos dados, alguns autores destacaram problemas relacionados à adequada digitação dos dados, o grande número de variáveis sem preenchimento, ausência de crítica a documentos incompletos ou inconsistentes vindo dos serviços hospitalares 29,30. Esses problemas comprometem a codificação da causa básica e a análise de variáveis demográficas, também importantes na caracterização da mortalidade infantil.

No componente "normatização e fluxo entre as esferas de gestão do SIM", cita-se, entre outros aspectos, a insuficiência no número de DO distribuídas, cujo cálculo da necessidade se baseia na própria notificação, gerando um círculo vicioso quando existe sub-notificação. Além disso, foram encontrados diferentes modelos de DO, inclusive em formatos não padronizados pelo Ministério da Saúde.

De um modo geral, a cadeia de problemas identificados na operacionalização do SIM teve como fulcro o componente "humano" na produção da informação de qualidade, sendo que esse perpassa todo o modelo, se reproduzindo na ausência de capacitação dos recursos humanos, de supervisão e controle, e a alta rotatividade de técnicos para o gerenciamento da informação em nível municipal. Essa percepção corrobora a abordagem informacional desenvolvida por Davenport 31 , onde a ênfase está no papel das pessoas em um determinado cenário em detrimento da tecnologia, buscando entender o modo pelo qual essas criam, distribuem, compreendem e usam a informação.

Entre os aglomerados de municípios estudados, verificou-se que houve indiscutível melhora na captação dos óbitos, de maneira geral, em relação ao quadriênio anterior, embora o patamar atingido pela pesquisa no ano 2000 não tenha se mantido. Uma das explicações possíveis é a alta 
rotatividade dos profissionais responsáveis pelo gerenciamento dos sistemas locais.

Uma contribuição adicional deste trabalho foi a construção de uma metodologia replicável em municípios com precariedade dos dados. A proposta considera dois eixos de atuação para a melhoria da operacionalização do SIM nesses municípios. O primeiro refere-se ao fortaleci-

\section{Resumo}

Esse estudo foi parte de uma pesquisa realizada em aglomerados de municípios brasileiros com precariedade das informações de óbito, visando à estimação da mortalidade infantil nessas áreas no ano 2000. O artigo discute as principais fontes de informação do óbito infantil nesses municípios, assim como alguns dos problemas relacionados à operacionalização do Sistema de Informações sobre Mortalidade (SIM). A metodologia adotada foi a de busca ativa de óbitos em fontes oficiais e não oficiais de informações previamente cadastradas. A busca ativa identificou 344 óbitos não processados no SIM (66\% do total de óbitos). Foi observado um baixo percentual de óbitos processados no SIM oriundos das fontes oficiais: cartórios e estabelecimentos de saúde, o que assinalou para problemas tanto na notificação do óbito e emissão da declaração de óbito quanto no fluxo de informações. A elevada contribuição do Programa Agentes Comunitários de Saúde/Programa Saúde da Família na identificação de óbitos infantis aponta para a necessidade de busca ativa de informações nesta fonte como rotina nos municípios com precariedade dos dados. A insuficiente capacitação dos profissionais e a alta rotatividade de técnicos foram questões-chave para a deficiente operacionalização do sistema.

Sistemas de Informação; Mortalidade Infantil; Programa Saúde da Família mento local da notificação de óbitos em fontes oficiais, como os estabelecimentos de saúde, cemitérios e cartórios. O segundo coloca a busca ativa em fontes alternativas, tais como o PACS/PSF e cemitérios não oficiais, como componente essencial. Transversalmente, enfatiza-se a capacitação permanente de recursos humanos.

\section{Colaboradores}

P. G. Frias, P. M. H. Pereira, C. L. T. Andrade e C. L. Szwarcwald contribuíram na concepção do artigo, análise dos resultados e redação final. P. G. Frias e C. L. T. Andrade participaram, também, da coleta de dados nos municípios.

\section{Agradecimentos}

Ao Ministério da Saúde pelo financiamento, à Fundação Nacional de Saúde (FUNASA), Secretarias de Estado e Municípios pelo apoio logístico ao trabalho de campo, e aos supervisores da pesquisa. 


\section{Referências}

1. Mello Jorge MHP, Gotlieb SLD, Soboll MLMS, Baldijão MFA, Latorre MRDO. O Sistema de Informações sobre Nascidos Vivos - SINASC. São Paulo: Centro Brasileiro para Classificação de Doenças; 1992.

2. Rede Interagencial de Informações para a Saúde. Indicadores e dados básicos para a saúde (IDB), 2006. http://tabnet.datasus.gov.br/cgi/idb2006/ matriz.htm (acessado em 16/Mai/2007).

3. Carvalho DM. Grandes sistemas nacionais de saúde: revisão e discussão da situação atual. Inf Epidemiol SUS 1997; 6:7-45.

4. Andrade CLT, Szwarcwald, CL. Desigualdades sócio-espaciais da adequação das informações de nascimentos e óbitos do Ministério da Saúde, Brasil, 2000-2002. Cad Saúde Pública 2007; 23:120716.

5. Szwarcwald CL, Leal MC, Andrade CLT, Souza Jr. PRB. Estimação da mortalidade infantil no Brasil: o que dizem as informações sobre óbitos e nascimentos do Ministério da Saúde? Cad Saúde Pública 2002; 18:1725-36.

6. Oliveira H, Pereira IPA. Estatísticas de mortalidade e nascidos vivos: considerações sobre os principais problemas. Inf Epidemiol SUS 1997; 6:15-9.

7. Associação Brasileira de Pós-Graduação em Saúde Coletiva. Pesquisa em saúde no Brasil: urgência do debate. Boletim da ABRASCO 2001; 79:1-2.

8. Centro de Informação Científica e Tecnológica, Fundação Oswaldo Cruz. Projeto para estimação da mortalidade infantil no Brasil no ano de 2000: relatório final. Rio de Janeiro: Centro de Informação Científica e Tecnológica, Fundação Oswaldo Cruz; 2002.

9. Secretaria de Vigilância em Saúde, Ministério da Saúde. 2a EXPOEPI Mostra Nacional de Experiências Bem-sucedidas em Epidemiologia, Prevenção e Controle de Doenças: relatórios das oficinas de trabalho. Brasília: Ministério da Saúde; 2003. (Série D. Reuniões e Conferências).

10. Fundação Nacional de Saúde. Manual de procedimentos do sistema de informação sobre mortalidade. Brasília: Fundação Nacional de Saúde; 2001.

11. Rede Interagencial de Informações para a Saúde. Indicadores básicos para a saúde no Brasil: conceitos e aplicações. Brasília: Organização Pan-Americana da Saúde; 2002.

12. Contrandriopoulos AP, Champagne F, Potvin L, Denis JL, Boyle P. Saber preparar uma pesquisa. 2a Ed. São Paulo: Editora Hucitec; 1997.

13. Champagne F, Denis JL. Análise da implantação. In: Hartz ZMA, organizadora. Avaliação em saúde dos modelos conceituais à prática na análise da implantação de programas. Rio de Janeiro: Editora Fiocruz; 1997. p. 49-88.

14. Yin RK. Discovering the future of the case study method in evaluation research. Eval Pract 1994 15:283-90.
15. Hartz ZMA, Champagne F, Contandriopoulos AP, Leal MC. Avaliação do programa materno-infantil: análise de implantação em sistemas locais de saúde no Nordeste do Brasil. In: Hartz ZMA, organizadora. Avaliação em saúde: dos modelos conceituais à prática na análise da implantação de programas. Rio de Janeiro: Editora Fiocruz; 1997. p. 29-48.

16. Frias PG. Análise de implantação do projeto de redução da mortalidade infantil em dois municípios de Pernambuco com semelhantes condições de vida [Dissertação de Mestrado]. Recife: Centro de Ciências da Saúde, Universidade Federal de Pernambuco; 2001.

17. Barreto IC, Pontes LK, Correa L. Vigilância de óbitos infantis em sistemas locais de saúde avaliação da autópsia verbal e das informações dos ACS. Rev Panam Salud Pública 2000; 7:303-12.

18. Mello Jorge MHP, Gotlieb SLD. O Sistema de Informação de Atenção Básica como fonte de dados para os Sistemas de Informações sobre Mortalidade e sobre Nascidos Vivos. Inf Epidemiol SUS 2001; 10:7-18.

19. Baggio MCR. A qualidade da informação de mortalidade em alguns municípios da Regional de Marília-SP: correção de distorções por meio de técnicas simples [Tese de Doutorado]. São Paulo: Faculdade de Saúde Pública, Universidade de São Paulo; 2002.

20. Façanha MC, Pinheiro AC, Fauth S, Lima AWDBC, Silva VLP, Justino MWS, et al. Busca ativa de óbitos em cemitérios da Região Metropolitana de Fortaleza, 1999 a 2000. Epidemiol Serv Saúde 2003; 12:131-6.

21. Pinheiro AMCM. Avaliação dos Sistemas de Informação de Nascidos Vivos e de Mortalidade para a obtenção da mortalidade neonatal em Ilhéus, Bahia [Tese de Doutorado]. São Paulo: Faculdade de Saúde Pública, Universidade de São Paulo; 2003.

22. Frias PG, Vidal SA, Pereira PMH, Lira PIC, Vanderley LC. Avaliação da notificação de óbitos infantis ao Sistema de Informações sobre Mortalidade: um estudo de caso. Rev Bras Saúde Matern Infant 2005; 5 Suppl 1:S43-52.

23. Almeida MF, Alencar GP, Novais HMD, Ortiz LP. Sistemas de informação e mortalidade perinatal: conceitos e condições de uso em estudos epidemiológicos. Rev Bras Epidemiol 2006; 9:56-68.

24. Ministério da Saúde. Importância dos sistemas de informações sobre mortalidade e nascidos vivos para os profissionais do Programa Saúde da Família. Brasília: Fundação Nacional de Saúde; 2001.

25. Secretaria de Vigilância à Saúde, Ministério da Saúde. Vigilância em saúde: dados e indicadores selecionados. Brasília: Ministério da Saúde, 2004.

26. Mello Jorge MHP, Gotlieb SLD, Andrade SM. Análise dos registros de nascimentos vivos em localidade urbana no Sul do Brasil. Rev Saúde Pública 1997, 31:78-89.

27. Tomé P, Reyes H, Piña C, Rodríguez L, Gutiérrez G. Características asociadas al subregistro de muerte en niños del estado de Guerrero, México. Salud Pública Méx 1997; 39:523-9. 
28. Jewkes R, Wood K. Competing discourses of vital registration and personhood: perspectives from rural South Africa. Soc Sci Med 1998; 46:1043-56.

29. Sarinho SW, Coutinho SB, Acioli TML. Mortalidade neonatal em Recife, PE em 1998: causas básicas e grau de conhecimento dos neonatologistas acerca do preenchimento das declarações de óbito. Pediatria (São Paulo) 2001; 23:279-84.
30. Vanderley LC, Arruda BKG, Frias PG, Arruda S. Avaliação da qualidade de preenchimento das declarações de óbito em unidade terciária de atenção à saúde materno-infantil. Inf Epidemiol SUS 2002; 11:7-14.

31. Davenport TH. Information ecology: mastering the information and knowledge environment. New York: Oxford University Press; 1997.

Recebido em 17/Set/2007

Versão final reapresentada em 19/Dez/2007

Aprovado em 19/Mar/2008 